\title{
Real-Time Etch-Depth Measurements of MEMS Devices
}

\author{
Sylvie Bosch-Charpenay, Jiazhan Xu, John Haigis, Peter A. Rosenthal and Peter R. Solomon \\ On-Line Technologies, Inc \\ East Hartford, CT 06108
}

\author{
James M. Bustillo \\ Berkeley Sensor \& Actuator Center, University of Califomia, \\ Dept. EECS, 497 Cory Hall, Berkeley, CA 94720-1770
}

\begin{abstract}
An in-situ, real-time process control tool was developed for MEMS deep-reactive-ion-etch (DRIE) fabrication. DRIE processes are used to manufacture high-aspect-ratio silicon structures up to several hundred microns thick, which would be difficult or impossible to produce by other methods. DRIE MEMS technologies promise to deliver new devices with increased performance and functionality at lower cost. A major difficulty with DRIE is the control of etch depth. Our research shows that it is possible to monitor the etch depth of various MEMS structures (holes, pillars, trenches, etc.) through measurement and analysis of the infrared reflectance spectrum. Depths as large as $150 \mu \mathrm{m}$ have been measured. Excellent correlation is found between the etch depths determined by analysis of these measurements and those measured with an SEM. In addition to etch depth, other parameters such as the photoresist thickness (e.g. mask erosion) can be simultaneously extracted. Based on these results, an infrared-reflectance etch monitor was integrated onto a reactive ion etcher at the Berkeley Sensor \& Actuator Center for real-time monitoring and end-point determination. The integrated optical metrology system demonstrated accurate real-time monitoring of the etch depth and photoresist mask erosion.
\end{abstract}

\section{INTRODUCTION}

DRIE processes are used to manufacture high-aspect ratio MEMS structures up to several hundred microns thick (see Figure 1). The resulting three-dimensional structures typically have 25-50 times the thickness of most surface-micromachined devices [1]. DRIE methods have the potential to yield advantages such as: 1) an improvement in $\mathrm{z}$-axis stiffness and robustness, $\mathrm{z}$ being the etch direction, 2) improved sensitivity, 3) increased robustness of devices to packaging stresses, 4) improvements in manufacturability, and 5) possibly lower cost. Examples of MEMS to benefit from DRIE high-aspect-ratio features include micro-inertial instruments (accelerometers, gyroscopes), for which the sensitivity can be increased by two orders-of-magnitude [2]; micro-disk-drive armatures, which can be produced with significantly lower cost and higher manufacturing throughput [3]; micro-assembly actuators, which have the required high out-ofplane and low in-plane stiffnesses [4], etc. Other DRIE applications include fabrication of SCALPEL mask membranes, requiring accurate and reproducible membrane thickness and buried-layer etch stops for thick silicon-on-insulator (SOI) designs.

While DRIE and other MEMS fabrication technologies promise new devices with increased performance and functionality at lower cost, one major difficulty is the control of etch depth during the DRIE process. Optical end-point detectors currently exist for DRIE of SOI wafers, but they require large open area test patterns $\left(300 \mu \mathrm{m}^{2}\right.$ area is typical) that do not etch at the same rate as the desired devices (which nominally have features sized in the micron range). For silicon (non-SOI) wafers, etch depth is measured using different systems depending on the pattern width, but always after the etch step is completed. Wide feature measurements are performed using profilometry or near-IR transmission measurements. For narrow structures (e.g., those less than $\sim 100$ $\mu \mathrm{m}$-wide) the measurements are performed using ex-situ destructive SEM. The lack of nondestructive metrology for narrow MEMS structures, as well as the need for in-situ process control, are the driving forces motivating this work.
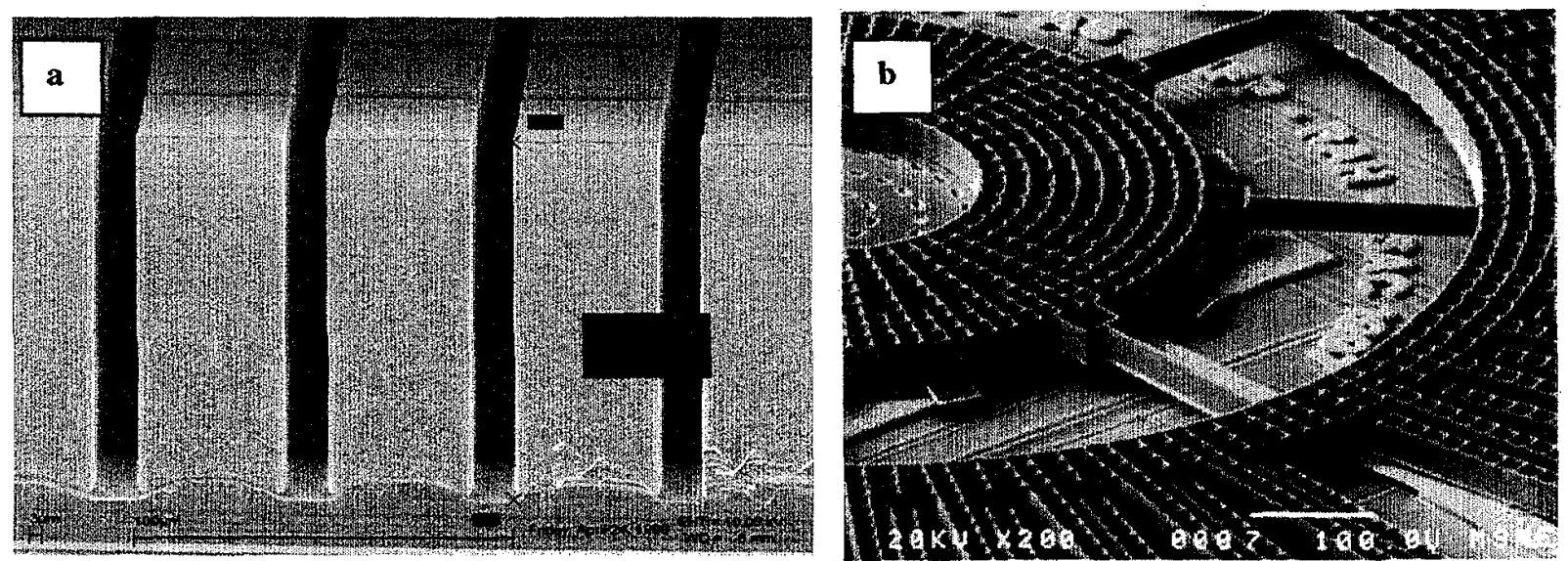

Figure 1. SEM photographs of a MEMS high-aspect-ratio, DRIE trench structure: a) cross-section of a grid of $10 \mu \mathrm{m}$-wide trenches whose depths have been measured by the FTIR method developed in this work, and which are the first step in the production of a 'Hexsil' micro-molded angular micro-actuator, b) finished $100 \mu \mathrm{m}$-thick angular actuator transferred and mounted onto a CMOS integrated circuit (Photos: Berkeley Sensor \& Actuator Center, University of California, Berkeley). 


\section{PRINCIPLE OF OPTICAL ETCH-DEPTH MEASUREMENTS}

When light reflects from a patterned and etched feature, interference occurs between those wavefronts returning from the top and the bottom of the feature. The resulting interference pattern contains information about the etch depth as well as the patterning masking layer. The analysis of such interference patterns in the visible spectrum has been used for the characterization of IC devices $[\mathbf{5 , 6 , 7 ]}$. We demonstrate that it is possible to make similar analyses with an infrared reflectance spectrum to measure the etch depth of MEMS trench structures.

Infrared reflectance of patterned MEMS structures - Figure 2 shows the infrared reflectance of MEMS 'Hexsil' structures with $10 \mu \mathrm{m}$-wide trenches forming an $\mathrm{x}-\mathrm{y}$. grid (similar to the structure shown in Figure 1a); the unetched areas are covered with photoresist. The reflectance in the spectral region $2-10 \mu \mathrm{m}(1000$ $5000 \mathrm{~cm}^{-1}$ ) was taken using an On-Line Technologies FTIR (Fourier Transformed Infrared) spectrometer at an incident angle of $4^{\circ}$ from normal, a resolution of $16 \mathrm{~cm}^{-1}$ and a measurement time of $<1 \mathrm{sec}$. Two types of interference fringes are evident in the spectrum; the large low-frequency fringe is caused by interference in the photoresist, while the small superimposed high-frequency fringes are caused by interference in the trenches.

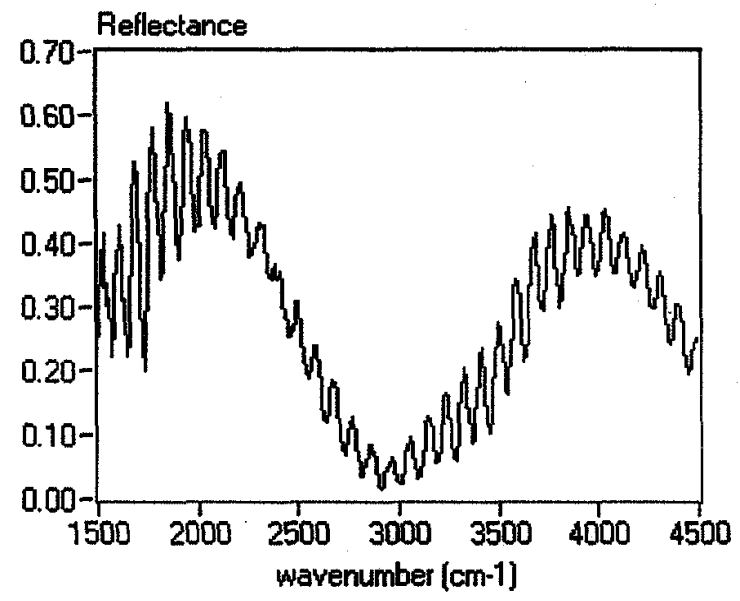

Figure 2. Infrared reflectance of MEMS 'Hexsil' structures with 10 um-wide trenches forming an $x-y$ grid. Unetched areas are covered with photoresist. The large, low frequency fringe is caused by interference in the photoresist, while the small, high frequency superimposed fringes are caused by interference in the trenches.

In order to extract parameters from the reflectance spectra of an etched structure, analysis models need to account for: 1) pattern effects, resulting in interference between wavefronts being reflected from different film stacks, and 2) thin film effects, resulting in interference between wavefronts reflected at various films interfaces.
Frequency analysis - For the case of a single material with etched regions (e.g. features etched in silicon without the photoresist masking layer), one can account for pattern effects by simply calculating the path difference, $\Delta x$, between the wavefront reflected by the unetched regions and the wavefront reflected by the etched regions. This relation can be expressed as: $\Delta x=$ $4 \pi h v / \cos \theta$, where $v=$ wavenumber of probing light $(v=$ $1 /$ wavelength), $h=$ trench depth, and $\theta=$ angle of incidence. Constructive interference will occur for two consecutive wavenumbers $v_{1}$ and $v_{2}$ when the path difference is equal to $2 \pi$ :

$$
\begin{gathered}
4 \pi h v_{1} / \cos \theta=4 \pi h v_{2} / \cos \theta+2 \pi \Rightarrow\left(v_{1}-v_{2}\right)=\cos \theta / 2 h \\
\Leftrightarrow \Delta v=\cos \theta / 2 h \Leftrightarrow F_{\text {trenches }}=2 h / \cos \theta \quad \text { (Equation 1) }
\end{gathered}
$$

with $F_{\text {trenches }}=$ fringe frequency in the reflectance spectrum. Equation 1 indicates a linear relation between fringe frequency and trench depth. A similar approach can be used to account for thin film effects and extract the thickness of a blanket film on a substrate [8]. In that case, the path difference is between the beams reflecting from the top and the bottom of the film, and the effect of the index of refraction of the film needs to be accounted for, which yields the following relationship for the fringe frequency $\mathrm{F}_{\text {film: }}$ :

$$
F_{\text {film }}=2 z n \cos \theta_{\mathrm{t}} \quad(\text { Equation 2) }
$$

with $z=$ film thickness, $n=$ film index of refraction, and $\theta_{\mathrm{t}}$ is the refracted angle with respect to normal where $\sin \theta_{t}=(\sin \theta) / n$.

In the case of MEMS structures, photoresist is present as a masking layer, and both pattern effects and thin film effects are present. A full spectrum fit (as opposed to a simpler frequency analysis) is usually required to simulate the compounding interference effects and extract etch-depth and film thickness [9]. However, in this case the interference patterns due to the photoresist film and that due to the trenches can be easily separated because they yield fringes of very different frequencies (see Figure 2). The different frequencies result from the very different values for trench depth (typically few tens of $\mu \mathrm{m}$ 's), and photoresist thickness (typically 1-2 $\mu \mathrm{m}$ ). As a consequence, Equation 1 was used to extract the etch depth using the high frcquency fringes, and Equation 2 was used to extract the photoresist thickness using the low frequency fringes. A more complete analysis including full spectrum fitting (useful for more complex film stacks) is given elsewhere [9].

Test of analysis model - Correlation between the etch depth asextracted by the FTIR method and measured by SEM were obtained for various structures and are displayed in Figures 3 and 4. Figure 3 shows the FTIR vs. SEM etch depth correlation for two types of MEMS trench structures, DRIE orthogonallypatterned 'Hexisil' micro-mold trenches $10 \mu \mathrm{m}$-wide and parallel trenches with lines and spaces $20 \mu \mathrm{m}$-wide. As seen in the figure, the FTIR-extracted etch depth was found to agree very well with SEM measurements. The residual photoresist thickncss is also plotted as a function of etch depth to show resist erosion (note: the photoresist thickness below $\sim 1 \mu \mathrm{m}$ is not plotted as the extracted thickness is not sufficiently reliable from the frequency analysis of Equation 2). 


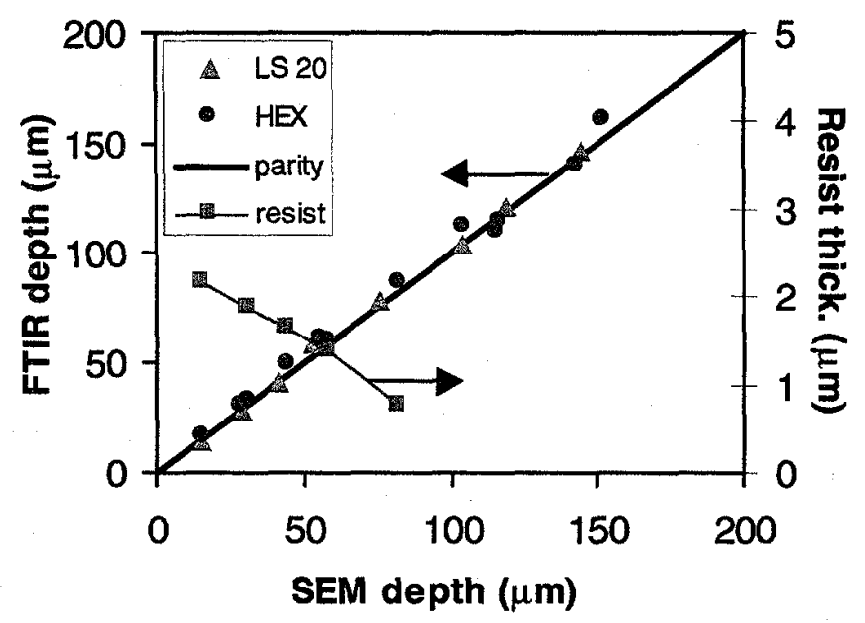

Figure 3. Correlation between the etch depth as extracted by the FTIR method to that measured by SEM. left axis: depths of orthogonally patterned 10 um-wide 'Hexsil' micro-mold trenches and 20 um-wide parallel trenches (lines and spaces), right axis: erosion of $2.5 \mu \mathrm{m}$ thick photoresist masking-layer thickness extracted by FTIR.

Influence of trench width - Figure 4 shows the FTIR vs. SEM depth correlation for etched parallel trenches with line and space widths of 4,8 , and $20 \mu \mathrm{m}$. As can be seen from the figure, the model applies linearly, as predicted, for large features $(20 \mu \mathrm{m})$, but requires an offset for smaller features ( 4 and $8 \mu \mathrm{m}$ ) - the offset magnitude depending on the feature width. This offset is thought to be due to trench edge effects, which may cause slightly different phase shifts, and results from the model limitations in the case of patterned features whose size are similar to the probing wavelength [10]. These limitations can only be eliminated by the use of

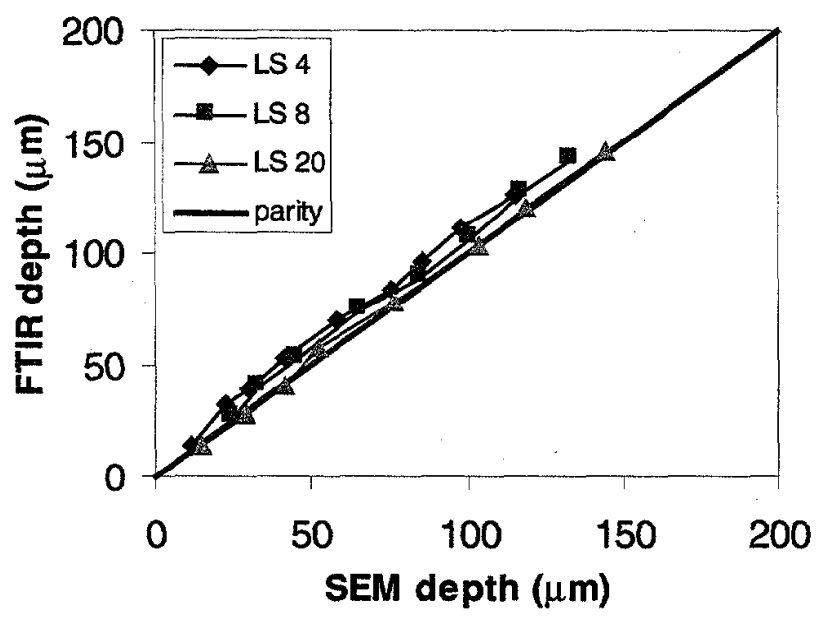

Figure 4. Correlation between the etch depth as extracted by the FTIR method to that measured by SEM for trench structures whose line/space ( $L S$ ) width were 4, 8 , and $20 \mu \mathrm{m}$ respectively. A trench-width dependent offset is observed for smaller trench widths. comprehensive electromagnetic vector-diffraction models for the exact simulation of the reflectance spectrum. Vector diffraction models solve the rigorous coupled-wave solution in that they account for all the interfering wavefronts [10]. The drawback of these models is that they are computationally intensive and usually require supercomputers or high-powered workstations [5], in contrast with the frequency model used in this work which has an extremely low computational load. Also, the vector diffraction models have been applied, so far, only to simple geometries which may not be relevant to MEMS applications.

The trench width limit at which the simple frequency model cannot be applied directly appears to be between 10 and $20 \mu \mathrm{m}$, as below these widths the correlation curves are progressively offset. However, the model can still provide good correlation with SEM, allowing an accurate determination of etch depth for narrow structures ( $4 \mu \mathrm{m}$ features being the smallest analyzed with the FTIR probe) when the offset is determined through a calibration procedure derived directly from data such as in Figure 4. As a consequence, the frequency model appears to be a satisfactory approach to MEMS etch depth extraction.

Influence of structures shape - The model has also been successfully applied to various MEMS structures having different geometrical patterns (holes, pillars, trenches, trench grids, honeycombs, etc.). The most complicated case was found to be that of parallel trenches (i.e., trenches organized as a diffraction grating), because the fringe frequency varied throughout the analyzed infrared spectrum. This behavior is consistent with the behavior of diffraction gratings, where it was found that the $0^{\text {th }}$ order diffraction efficiency varies with wavelength in an oscillating manner, and can only be accurately simulated by vector diffraction analysis [10]. Other geometrical patterns did not display this behavior, presumably because of their more isotropic arrangement (e.g. orthogonally-patterned 'Hexsil' micro-molds). In spite of these $0^{\text {th }}$-order deficiencies it was observed that even for the parallel trench samples the fringe frequency, averaged over the whole IR spectrum, correlated very well with the etch depth measured by SEM (see Figure 4) - still allowing accurate etch depth extraction.

\section{In-Situ, Real-Time Etch-Depth Measurements}

The FTIR sensor has been integrated on a Surface Technology Systems (STS) Multiplex ICP deep reactive ion etcher at the Berkeley Sensor \& Actuator Center at the University of California at Berkeley. Real-time measurements of etch depth and photoresist thickness (e.g. photoresist mask erosion during etch) were obtained on MEMS structures (as in Figure 1a). Etch depth as a function of etch time for this structure is shown in Figure 5. The etch process used was the switched Advanced Silicon Etch (ASE) process [11]. The models from Equation 1 and Equation 2 were used to extract the etch depth (using FTIR analysis of the fast-varying superimposed fringes), and the eroding photoresist thickness (using FTIR analysis of the slow-varying fringes). The single-point measurement time, which includes the FTIR reflectance measurement and analysis, is approximately $1 \mathrm{sec}$. The measurements are precise enough (when measuring depth) to see the sequential "passivating" and "etching" steps that are characteristic of the switched ASE process. The result is clearly 


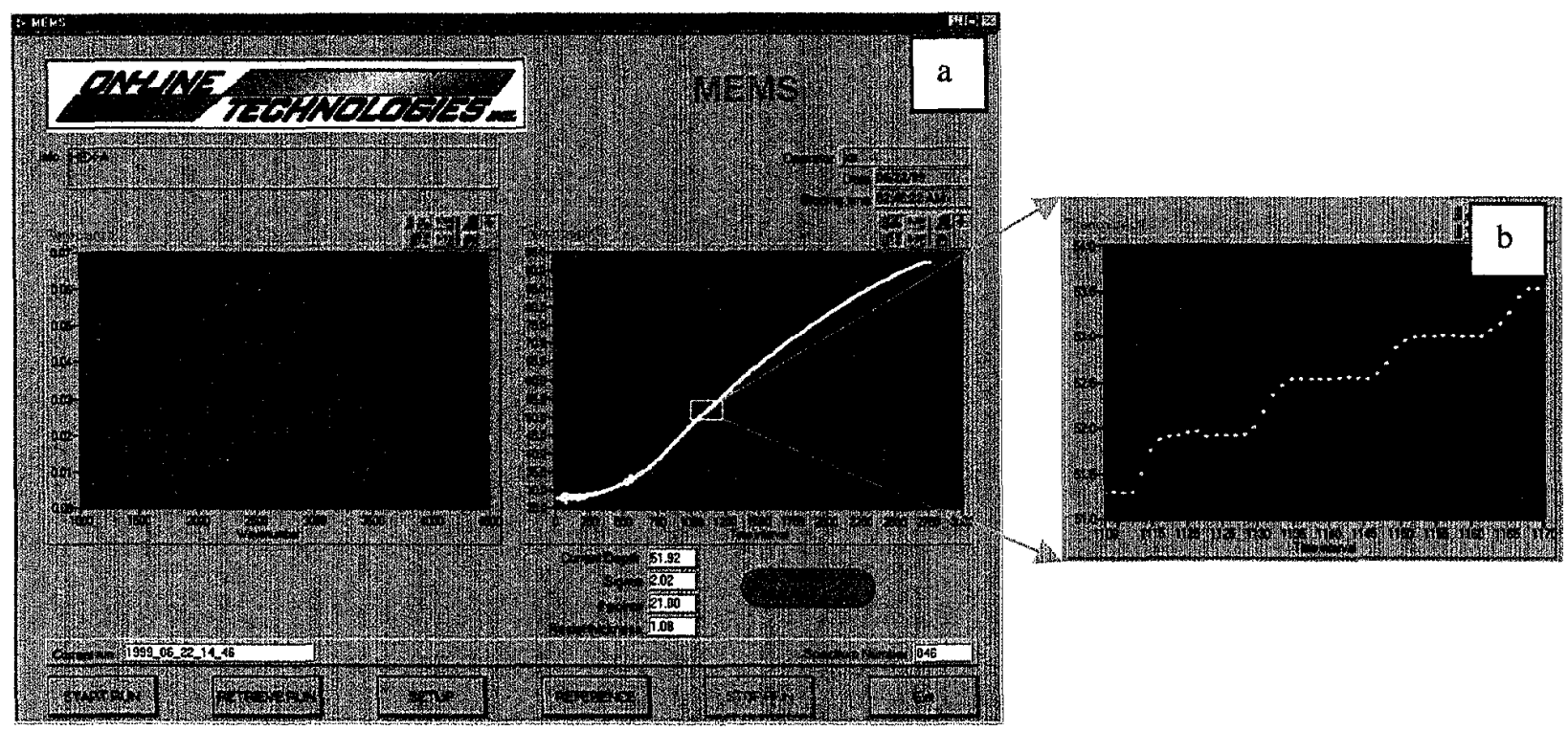

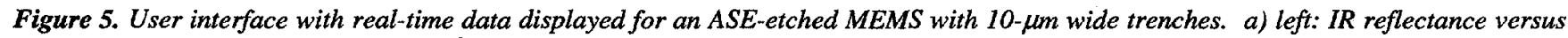
wavenumber $\left(v=1 /\right.$ wavelength) in $\mathrm{cm}^{-1}$, and right: etch depth $(\mu \mathrm{m})$ versus time (sec.) for a $92 \mu \mathrm{m}$ trench. b) etch depth versus time with an expanded scale, clearly displaying the sequential etch and passivation layer deposition steps typical of the ASE process. The typical switching period is 10-20 sec.

Table 1. Summary of the sensor's capabilities.

\begin{tabular}{|l|l|}
\hline MEMS structures which can be measured & $\begin{array}{l}\text { Pillars, holes, trenches, trench grids, honeycombs, of } \\
\text { etched width less than } \sim 100 \mu \mathrm{m}\end{array}$ \\
\hline Etch depth precision (reproducibility) & $0.05-0.5 \mu \mathrm{m}$ depending on the structure \\
\hline Etch depth accuracy (as compared to SEM) & $\sim 1-2 \mu \mathrm{m}$ (estimated) \\
\hline Maximum measurable etch depth & $>200 \mu \mathrm{m}$ \\
\hline Current spot size & $\sim 800 \mu \mathrm{m}$ DIA \\
\hline Measurement time & $\sim 1 \mathrm{sec}$. \\
\hline Measurement spot range & 2 \\
\hline Probing IR wavelength & $\pm 5 \mathrm{~mm}$ in both $\mathrm{x}$ and $\mathrm{y}$ direction \\
\hline
\end{tabular}

defined staircase-like etch-depth vs. time profile. Table 1 displays a summary of the integrated optical metrology system's capabilities.

\section{CONCLUSIONS}

In this work in-situ real-time DRIE etch depth and eroding photoresist thickness measurements were obtained on MEMS devices using infrared reflectance analysis. To our knowledge, this is the first time that these measurements have been made while DRIE plasma-etching MEMS structures. The method is nondestructive and provides real-time process analysis. Better realtime in-situ monitoring of MEMS processing will allow improved process accuracy and controllable process repeatability. The specific anticipated benefits of the new technique are: 1) to reduce cost through the reduction of destructive measurements and the improvement in process control, 2) to increase the reproducibility of the MEMS structures through better process control, 3) to provide useful feed-back for process development, thus reducing development time.

\section{ACKNOWLEDGEMENTS}

This work has been done as a collaboration between On-Line Technologies of East Hartford CT, the Berkeley Sensor \& Actuator Center (BSAC) at the University of California at Berkeley and Surface Technology Systems (STS) of Newport, Gwent UK, with funding from the National Science Foundation (NSF), contract DMI-9860514. Fabrication and microscopy work was done at the Microfabrication Laboratory in the Electrical Engineering \& Computer Science Department also at U.C. Berkeley.

\footnotetext{
${ }^{1}$ Optical diffraction limits preclude spot sizes smaller than $\sim 400 \mu \mathrm{m}$. MEMS devices smaller than this minimum spot size can however still be measured provided they are geometrically isolated from other DRIE etched features.

${ }^{2}$ Translation range of infrared spot over sample is strictly related to equipment geometry at the etch chamber - in this case a small overhead viewport into the STS Multiplex ICP system.
} 


\section{REFERENCES}

${ }^{1}$ J. Bhardwaj, H. Ashraf, A. McQuarrie, "Dry silicon etching for MEMS", Proc. of the Third International Symposium on Microstructures and Microfabricated Systems, Montreal, Canada, (1997), pp. 118-130.

${ }^{2}$ T. Juneau, M.A. Lemkin, T.A. Roessig, W.A. Clarck, R.T. Howe, J.M. Bustillo, T. Brosnihan, A.P. Pisano, "Commercialization of precision inertial sensors with integrated signal conditioning", Sensors Expo, May (1998).

3 D.A. Horsley, A. Singh, A.P. Pisano, R. Horowitz, "Angular micropositioner for disk drives", Proc. of the IEEE Micro-ElectroMechanical Systems Workshop, Nagoya, Japan, (1997), pp. 454.

4 C.G. Keller, R.T. Howe, "HexSil tweezers for teleoperated micro-assembly", Proc. of the IEEE Micro-Electro-Mechanical Systems Workshop, Nagoya, Japan, (1997), pp. 72.

s M.E. Lee, C. Galarza, W. Kon, W. Sun, F.L. Terry, Jr., "Analysis of reflectometry and ellipsometry data from patterned structures", Characterization and metrology for ULSI Technology: 1998 International Conference, Seiler D.G. et al. Editors, the American Institute of Physics, Pub., (1998).

6 P.A. Heimann, R.J. Schultz, "Optical etch-rate monitoring: computer simulation of reflectance", J. Electrochem. Soc.: Solid State Science and Technology, Vol. 131. No. 4, (1984).

7 M. Haverlag, G.S. Oehrlein, "In-situ ellipsometry and reflectometry during etching of patterned surfaces: experiments and simulations", J. Vac. Sci. Technol., B 10 6, (1992).

${ }^{8}$ E. Hecht,. "Optics", Addison-Wesley Pub. (1987).

9 S. Bosch-Charpenay, J. Xu, J. Haigis, P.A. Rosenthal, P.R. Solomon, and J.M. Bustillo, "Real-Time Etch-Depth Measurements of MEMS Devices" submitted to the IEEE Journal of Microelectromechanical Systems (2000).

${ }^{10}$ D.A. Gremaux, and N.C. Gallagher, "Limits of scalar diffraction theory for conducting gratings", Applied Optics, Vol. 32, No. 11 (1993).

${ }^{11}$ F. Larmer, A. Schilp, "Method of Anisotropically Etching Silicon", German Patent DE4241045. 